\title{
A Virtual Environment Designed To Help Students Understand Science
}

\author{
Mark Windschitl, Bill Winn \\ University of Washington, College of Education, 115 Miller Hall Box 353600, Seattle WA, 98195 \\ Tel: 206-221-4736 Fax: 206-543-8439 \\ E-mail: mwind@u.washington.edu, billwinn@u.washington.edu
}

\begin{abstract}
This paper reports research in progress regarding the effectiveness of immersive virtual environments (IVEs) in helping students understand complex scientific concepts and principles. The subject matter is the ecology of Puget Sound, in Washington State. The purpose of the project is to determine how different characteristics of immersive virtual environments are exploited by learners to improve their understanding of complex environmental processes, and to determine what aspects of the virtual environment facilitate or hinder this understanding. In this paper we describe: The conceptual framework that is guiding our work; the IVE itself, Virtual Puget Sound (VPS); the tasks students perform in it; and, the research that is currently underway. We conclude with some observations about the general role of IVEs in learning science.
\end{abstract}

Keywords: virtual reality, science education, visualizations, higher education

\section{Conceptual Framework}

Our work is guided by a theoretical framework built from three interwoven strands: Learning in IVEs, knowledge construction, and conceptual change in science.

\section{Learning in IVE's}

Research to date (Byrne, 1996; Dede, Salzman, \& Loftin, 1996; Winn \& Jackson, 1999) has suggested that IVEs have a number of properties that help students understand scientific concepts and principles. An important feature of immersive virtual reality is the radically different interface to the computer, which can quickly become transparent to the user (Bricken, 1991). Rather than using a keyboard and mouse view output on a monitor, the student interacts with the computer by gesture or speech, and views data on miniature screens inside a helmet whose orientation is tracked and responded to in real time by the computer. This gives learners a sense of immersion because they can visually survey in the IVE just as in the real world, and can manipulate objects in a quasi-natural fashion by touching them, picking them up and placing them elsewhere. Dede (1995) has pointed out that this allows a much more direct experience of concepts and principles, removing the burden of having to learn a complex and abstract symbol system first. Also, because every object in an IVE is constructed from data, students can observe and interact with objects that have no physical or perceptible form in the real world in the same way they interact with real objects. The advantages of seeing and handling objects are thus extended to phenomena like atomic structure (Byrne, 1996), the movement of nitrogen through the nitrogen cycle (Osberg, 1997), or the effect of destroying forests on global warming (Jackson, Taylor, \& Winn 1999). The manipulation of objects, movement in three dimensions and real-time computer responses enhance the feeling of "presence" -- the sense that you are "in" the IVE rather than in the classroom wearing headgear (Winn et al., 1997).

\section{Knowledge Construction}

These and other properties of IVEs make them ideal places to implement constructivist principles of learning (Dede, 1995). Our research, and the IVEs we build, assume that students actively construct understanding for themselves by observing, experimenting, and interpreting the dynamics of any environment rather than by simply "taking in" information provided by the teacher or computer. This knowledge construction is enhanced within the framework of a problem-solving experience (Windschitl \& Andre, 1998). Problem-solving furnishes both a purpose for exploring a domain and a meaningful context to guide and organize experience. However, student activity in an IVE must be scaffolded and the consequences of actions must be immediately and clearly shown or the student can wander aimlessly through the IVE and learn little (see Rose, 1996, for how this can be avoided). 


\section{Conceptual Change}

Given the properties of IVEs that can support constructivist approaches to learning, our research into how IVEs help students understand science is guided by conceptual change theory (Thorley \& Stofflet, 1996; Vosniadou, 1994). In particular, we refer to a model of conceptual change adapted from Posner et al. (1982) that requires: 1) students' observations to lead to inconsistency with currently-held beliefs about how the world works, 2) students propose a plausible explanation (hypothesis) to explain the inconsistency, 3) this hypothesis is intelligible to the user, 4) the hypothesis is tested in the IVE and successfully explains observed phenomena, 5) this process takes place within an active, problem-solving experience. These strategies require that our immersive virtual world represent, directly or metaphorically, representations of natural phenomena that are consistent and scientifically valid. They also require that students can test hypotheses by controlling some variables while manipulating others, and that the results of these operations are visibly or audibly evident in the IVE that surrounds them.

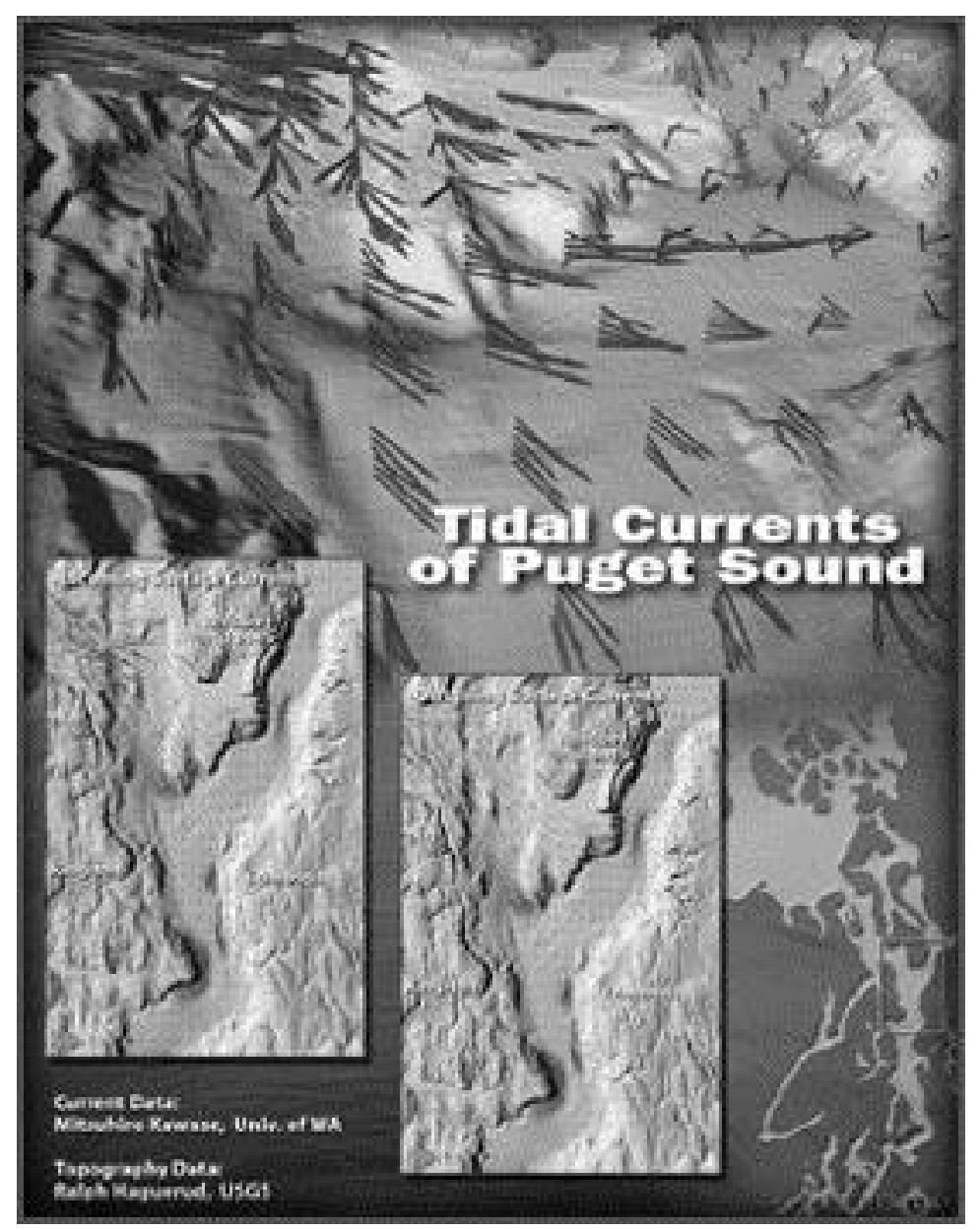

Figure 1. Graphic capture from Virtual Puget Sound

\section{Virtual Puget Sound (VPS)}

Educators and scientists at the University of Washington have embarked on an ambitious project aimed at uniting the vast databases of several different natural science domains and creating a unified model of Puget Sound. Referred to as the PRISM project (Puget Sound Regional Synthesis Model-- http://www.prism.washington.edu), the goal of PRISM is to integrate demographic, atmospheric, hydrographic, and oceanographic databases and models of the region to describe and predict environmental phenomena. From these databases (and in particular from an adaptation of the Princeton Oceanographic Model of water circulation; Blumberg \&. Mellor, 1987), scientific data 
has been transformed into two-dimensional visualizations. These complex visualizations have then been rendered as three-dimensional "spaces", object, properties and events to form Virtual Puget Sound (see Figure 1).

Virtual Puget Sound simulates water movement for a complete tidal cycle (roughly 24 hours), sampled at 30 minute intervals, at twelve depths, on a 300 by 600 meter grid covering the whole of Puget Sound. Water speed and direction are shown as dynamic 3-dimensional arrows, whose length represents water velocity and which point in the direction the current is moving. Salinity is shown as stacked spheres, one sphere for each of twelve depths, whose color represents the amount of salt in the water in parts per thousand. The least salty water is shown as blue, the saltiest as red. These representations may be turned on and off at will. They may also be animated at any speed, or frozen so that students may make measurements while controlling for changes over time. Students may fly, in three dimensions, to any point in the environment, using simple gestures and a push-button. A "God's-eye" view of the entire Sound, with the model animated and looping at, for instance, 20 steps a second, shows large scale tidal movement of the water in and out of the Sound in a way that is inaccessible by direct observation or instrumentation.

In this IVE, students may make measurements at any of five special "stations" around the Sound, selected because they show the range of relevant characteristics of the Sound. Stations are equipped with virtual tools for measuring current speed, current direction and salinity, as well as for changing depth in the water column. Students can also turn the tidal cycle on and off and can control its speed. They see a graphic representation of the tidal profile for that site and may move to any point in the tidal cycle by dragging a cursor over the graph, to high water or to halfway between high and low tide, for example. The arrows representing current speed and direction move as learners manipulate the tidal cycle directly in this way.

Students interact with the virtual tools for navigation, manipulation, and measuring in a number of ways. Some actions are initiated through a push-button menu which may be summoned and then "floats" within easy reach. The student touches items on the menu to turn the representations of current or salinity on and off, to fly directly to each of the five stations, or to visit bird's-eyes and God's-eye views of the Sound. The tools at each station are controlled by touching a push button, or by grabbing them. The students control their depth in a station by moving a slider up and down a depth scale. They measure salinity and the speed and direction of the current by selecting buttons on a data panel. Preliminary data show that the interface is fairly intuitive and that the tools can be learned after brief practice.

\section{Learning Activities}

Our research is intended to identify those properties of IVEs that contribute significantly to students' understanding of complex environmental concepts and principles. The activities students perform in Virtual Puget Sound must therefore meet two criteria: They must get students to do things that would be difficult to do in a twodimensional, non-immersive environment, such as view the world and reason about it in three dimensions from different points of view; and, the activity must prompt students to explore the world in a problem-solving task that brings them into contact with conceptual phenomena that may be at odds with their existing understandings. In one scenario, for example, students must decide where Metro (authorities from the three counties around Seattle) should build a new sewage treatment plant that will dump treated effluent into Puget Sound. The students start by making observations of current speed and direction, and salinity from a variety of vantage points, above and below water, and make measurements at some or all of the five stations. What they observe may or may not be at variance with their pre-existing conception of how water moves in the ocean (preliminary data show that even oceanography undergraduates have important misconceptions about this topic). They propose a hypothesis about where it would be best to place the effluent pipe and test that hypothesis by making measurements at that location at different times in the tidal cycle. In a second scenario (the one used in this study) students use problem-solving strategies similar to those described above to identify the most likely habitat for a voracious species of fish that has been observed for the first time in Puget Sound.

Before entering the IVE, students watch a videotape that orients them to the shape, topography and bathymetry of Puget Sound. This includes a segment that shows scientists on a research vessel collecting temperature, salinity, and current speed samples from Puget Sound. The tape also shows how scientists use these data to construct models and that these models are then used to create two-dimensional and three-dimensional 
visualizations. The purpose of the video is to establish a sense of authenticity and meaningfulness regarding the phenomena they will experience in the virtual world. Students then receive training in the use of the interface and the tools they will have to use. When they have achieved competency in these skills, they are given the problem to solve, (where the voracious fish might most likely be found). The extent to which they receive further guidance, either before they enter the IVE or while they are in it, is a factor that we attempt to control through strict protocols about what research assistants can and can not say to the participants. Other studies (Jackson et al., 1999) have shown that some students require considerable assistance with tasks in IVEs.

\section{On-Going Research}

This first round of research is concerned with whether VPS and its problem-solving activity frame can be used to remediate alternative conceptions in learners. The initial research is also looking at the way students interpret specific representations of phenomena, how successfully they use the tools and how they generally react to the interface. These latter aspects of the research are qualitative in nature. Our research is intended to find those properties of IVEs that are most likely to improve understanding of natural processes. In the next round of research, we will begin by manipulating two factors that are likely to affect understanding in immersive virtual environments: The extent of a subject's freedom to observe and move in three dimensions; and, the extent and nature of interaction with objects in the IVE. Earlier research has shown that the "naturalness" of both of these activities improves the student's sense of presence in the IVE (Bricken, 1991; Winn et al., 1997). We manipulate the former, for example, by allowing one group of subjects complete freedom to move in three dimensions while constraining a second group to visit only the five stations and having a third group work with a non-immersive desktop version of the IVE. We manipulate the latter by having one group of students interact with the objects in the IVE and having another watch as the manipulations occur automatically. We also vary the metaphors we use for salinity (colored spheres or animated iso-surfaces) and for current speed and direction (arrows showing vectors, or "worms" that leave traces of where they have been). In addition, we vary the tasks that students must perform, so that some of the time they are conducting activities that are likely to be easier in an IVE and the rest of the time they are not. For example, understanding how water movement is affected by changes in density, brought about by the relative movement of bodies of water of different salinity, is likely to be easier inside a IVE. Viewed from an angle above the water, the salinity spheres can be observed changing color in time with the tidal cycle, and a salinity probe placed in the water column can indicate whether the saltiest water is above or below less salty water. On the other hand, the tidal cycle itself - two high tides and two low tides per cycle - is much more readily inferred from observations of the movement of the sea surface, which can easily be shown in two dimensions on a flat screen.

\section{Subjects}

We are working initially with university undergraduates in introductory courses in Oceanography and related sciences. Later, we plan to take immersive and non-immersive versions of VPS to high schools and middle schools. Indeed, we have already tested a module based on the sewage scenario, using non-immersive materials, in a grade eight science class.

\section{Procedures and Dependent Measures for the Current Study}

Subjects begin by completing a pretest designed to assess their understanding of water movement in Puget Sound and to gather relevant demographic information. They then view a videotape that provides an orientation to how oceanographic data is collected and transformed first into visualizations and then into a virtual world. Students then receive a brief think-aloud training. Because our research is concerned with conceptual change, we are particularly interested in the reasoning students engage in while performing their tasks. For this reason, students are trained to think aloud while in the IVE. Analysis of these protocols provides a rich source of information on how students' ideas about the environment change as they interact with it in real time. These verbal protocols also help us identify any new misconceptions that might arise as a result of working in a virtual environment rather than the real one.

Research assistants then give participants a 30-minute, in-the-world training for navigation and tool use. Students are then given the problem to solve and are briefed in varying amounts of detail on how to go about finding a solution. Once inside the IVE, a computer file saves every action they perform with a time stamp so that their session can be recreated afterwards. A video record is kept of each session, both of what each student sees and does in the IVE (a video feed from one eye in the helmet is taped) and of the students' actions, seen from outside the IVE. 
Students take a posttest (parallel to the pretest), are debriefed and interviewed. We use stimulated recall to help students explain why they did things in a particular way when we notice sequences of actions that may be especially revelatory.

\section{Findings}

To date, approximately 20 students have participated in a pilot study. We have attempted to evaluate the qualities of the virtual world in conjunction with examining what and how students learn in Virtual Puget Sound. Students spent an average of 90 minutes in the world (30 minutes of training and 60 minutes of problem-solving). T-tests comparing pre- and posttests reveal modest, but statistically significant gains in conceptual understanding of Puget Sound water phenomena (pretest mean $=10.06$ [SD 3.05], posttest mean=12.06 [SD 3.83], $\mathrm{p}<.046$ ). Interestingly, the VPS experience apparently generated serious misconceptions about the relationship between salinity and water depth. Twenty percent of the students, who originally held the correct conception that more saline water settles below fresher water, switched to the reverse conception after their experiences in VPS. Students varied dramatically in their understanding of the concepts presented in the world, their understanding of the way properties were represented, and their ability to solve the problem. About one-third of the students expressed a deep sense of confusion about what the water current arrows meant, not being able to remember what the length of the arrows signified or failing to make sense of their color. One student mistakenly thought that the color of the arrows represented salinity, and this condition by itself profoundly (negatively) affected her ability to make sense of the environment. We discovered this only during the posttest interview. The student stated:

Honestly, I couldn't tell you [about the speed] apart from the pink color. And I think if I wasn't looking for the pink I would have had a better sense for the speed but since I was, since the glasses I had on was looking for the pink, it made it difficult for me to watch for things at the same time and separate them out.

Also, students who "saw the same phenomena" made radically different interpretations of it. Water coming into Puget Sound tend to speed up as it passes through narrow straits-- a phenomena generalizable to any type of fluid flow. One student made a completely contradictory conclusion:

I can tell you what I would have said before and then right now. I would have said before that the water moves faster in the narrower channels. And that's not what I think the model suggested. The model suggested that the closer you get to the ocean, the open water, the faster the water moves. And I know nothing about water so you've got a good subject.

Another student exhibited a more scientifically "correct" conclusion:

Yeah, so the shapes...the things that it would...I'm thinking it's like airflow where it bumps into things, stops, gets turbulent, whatever. One of the things I was kind of looking at was--okay-here's narrowing, and sure enough it did tend to speed up where it was constricted. Now I don't know water, but air does that.

One consistent response (from $\sim 80 \%$ of students) was that after the VPS experience, they had a much deeper appreciation of the complexity of water movement in Puget Sound. Water can indeed move in different directions and at different speeds depending on the depth at which these measures are taken. The general velocity of the current differs with time in the tidal cycle and with the narrowness of the channels it passes through. Although only a small number of students were able to put into concrete terms their new mental models of water movement, the appreciation of the complexity of water movement that was exhibited by almost all students was a significant finding.

This pilot has convinced our research group of one thing in particular: that the learning that occurs in immersive virtual environments is highly dependent upon the complexity and character of the problem that learners are asked to solve, and, learners' interpretations of visualizations that represent certain phenomena (i.e. magnitude of 
current represented by both color and length of arrows, salinity represented by colors of spheres at different depths). Our experiences to date lead us to conclude that generalizations about learning in IVEs are difficult to make because learners' experiences are so varied, so dependent on the specific character of 1) visual representations, 2) the complexity of the underlying phenomena, and 3) the kind of structured experiences learns can have in these worlds.

Clearly this kind of basic research is necessary to reveal trends in how individuals use IVEs and to establish "proof of concept" that individuals can learn in virtual worlds; however, in a practical sense, virtual world experiences are of such brief duration they are limited in what they can tell us about conditions that would maximize learning. These optimal conditions, of course, would include 1) more extended opportunities for individuals to use the IVE, 2) the opportunity to have sense-making dialogue with others in between these experiences, and 3) embedding the use of IVEs in longer term curricular frameworks that include hands-on activities, readings, reflection, and some direct instruction.

\section{VR and Science Learning}

The results of this project will identify how learners use IVEs to make sense of complex natural phenomena. More important perhaps, they will indicate when it is not advantageous to use this technology. The project also provides guidelines about technologies and strategies for interacting with IVEs. We also expect that virtual reality will be most useful for learning science when problems are best solved by three-dimensional spatial reasoning and when it is an advantage to change viewpoint. We also expect this will be true when the student's physical position in a IVE is itself meaningful. For example, physically changing depth in the water column and directly observing what occurs while depth is changing carries with it advantages for learning about the marine environment. More detailed analyses of this study and additional studies are now underway to answer several of the questions posed in this paper-- their results will contribute to our understanding of how learners use IVEs to understand complex science concepts.

\section{References}

Blumberg, A. F. \& Mellor, G. L. (1987). A description of a three-dimensional coastal ocean circulation model. In N. Heaps (Ed.), Three-Dimensional Coastal Ocean Models, 208 pp., American Geophysical Union.

Bricken, M. (1991). Virtual worlds: No interface to design. In M. Benedikt (Ed.), Cyberspace: First steps. Cambridge, MA: MIT Press.

Byrne, C.M. (1996). Water on tap: The Use of Virtual Reality as an Educational Tool. Ph.D. Dissertation, department of Industrial Engineering, University of Washington, Seattle, WA.

Dede, C. (1995) The Evolution of Constructivist Learning Environments: Immersion in Distributed, Virtual Worlds. Educational Technology, 35 (5), 46-52.

Dede C. J., Salzman, M., \& Loftin, R.B. (1996). The development of a virtual world for learnin Newtonian mechanics. In Brusilovsky, P., Kommers, P., \& Streitz, N. (Eds.), Multimedia, Hypermedia, and Virtual Reality: Models, Systems and Applications. Proceedings of the First International Conference, MHVR '94. Berlin: Springer Verlag.

Jackson, R., Taylor, W., \& Winn, W. D. (1999, April). Peer collaboration in virtual environments: An investigation of multi-participant virtual reality applied in primary science education. Paper presented at the annual meeting of the American Educational Research Association, Montreal.

Osberg, K. M. (1997, March). The effect of having grade seven students construct virtual environments on their comprehension of science. Paper presented at the annual meeting of the American Educational Research Association, Chicago, Il.

Posner, G. J., Strike, K. A., Herson, P. W., \& Gertzog, W. A. (1982). Accommodation of scientific conception: Toward a theory of conceptual change. Science Education, 66 (2), 211-227.

Rose, H. (1996). Zengo Sayu: An Immersive Educational Environment for Learning Japanese. HIT Lab. Technical Report, R-96-6. Seattle, WA: Human Interface Technology Lab.

Thorley, N., \& Stofflet, R (1996). Representation of the conceptual change model in science teacher education. Science Education, 80 (3), 317-339.

Windschitl, Mark and Andre, Tom, 1998, Using computer simulations to enhance conceptual change: The roles of constructivist instruction and student epistemological beliefs. Journal of Research in Science Teaching, 35 (2), 145-160. 
Vosniadou, S. (1994). Conceptual change in the physical sciences. Learning and Instruction, 4 (1), 45-69.

Winn, W. D., Hoffman, H., Hollander, A., Osberg, K., Rose, H., \& Char, P. (1997, March). The effect of student construction of virtual worlds on the performance of high and low ability students. Paper presented at the annual meeting of the American Educational Research Association, Chicago, IL.

Winn, W. D., \& Jackson, R. (1999). Fourteen propositions about educational uses of virtual reality. Educational Technology, 39 (4), 5-14.

\section{Acknowledgments}

This project is supported through a grant to the first author from the National Science Foundation's program Research on Educational Policy and Practice No. 9873620. I would like to acknowledge research assistants Ruth Fruland, Nick Hedley, Lori Postner, and Angela Thomson-Bulldis for their efforts and guidance in helping conduct this study. 